\title{
Tracking Eyes in Service Prototyping
}

\author{
Monchu Chen ${ }^{1}$ and Veraneka Lim ${ }^{1,2}$ \\ ${ }^{1}$ Madeira Interactive Technologies Institute, Portugal \\ ${ }^{2}$ Eindhoven University of Technology, Netherlands \\ monchu@m-iti.org, v.limatue.nl
}

\begin{abstract}
A mobile eye tracker was used to collect viewing behavior in a mixed reality immersive Cave Automatic Virtual Environment (CAVE) environment to evaluate a design concept of a tourist information office. The synthetic office consists of physical artifacts and virtual contents projected onto three walls of a room-sized cube. A Think Aloud study was conducted with both a goal-oriented condition and a free-browsing condition while subjects wearing the eye-tracker. Multiple Augmented Reality markers were used to reconstruct gaze positions in the coordinate system of the real environment. Gaze points were later aggregated to create heat maps, which were used as textures for a computer 3D model replication of the synthetic tourist office. The interactive visualization of the 3D heat map showcases different viewing patterns for different conditions. The insights suggest the combination of eye-tracking and mixed reality environment to be a valuable tool for prototyping service design of similar kinds.
\end{abstract}

Keywords: Eye-Tracking, Service Design, Experience Prototyping, Heat Map.

\section{Introduction}

Eye-tracking technique has been applied to various areas such as psychology, human factors, marketing research, etc. It has been particularly successful in the usability evaluation of human-computer interface design [1]. An eye-tracker allows researchers and designers to follow the eye gaze of a user, providing insights to how a user perceive and interact with an interactive system. Knowledge about when and where customers target their visual attention and how this is affected by certain aspects of the service or environment is expected to aid service designers in understanding customers' mental processes under circumstances like information processing and its influence on decision making. Several eye movement analysis metrics have been proved to be useful for desktop scenarios (e.g. a web site design), however, little have been applied to ubiquitous computing environments (e.g. a service design). Therefore, this paper proposes to depict customer experiences while using a service through the study of eye gaze.

In the last decades, most research on visual attention was primarily based on twodimensional (2D) stimuli and static scenarios, regarding both observer and content (e.g. websites and interfaces). With the increased interest in interactions of people in 
natural settings and mobile uses of interaction technology, current advancements in mobile eye tracking systems now allows the observer to freely interact physically in a three-dimensional (3D) scenario, providing the means to assess eye gaze and visual attention and understand mental processes in arbitrary 3D environments (e.g. driving simulator and virtual reality). This approach answers questions on how to improve the guidance of visual attention afforded by 3D environments with multiple levels of depth. Research on visual attention, however, has still mainly targeted 3D objects and $3 \mathrm{D}$ virtual reality [2, 3, 4]. Limited to none research has focused on visual attention in Service Design, which is a new, holistic, multi-disciplinary and integrative field [5]. One reason why eye tracking hasn't been applied to Service Design might be related with the issue of eye gaze data collection and representation in $3 \mathrm{D}$ environments for an effective interpretation of eye tracking results.

A common technique for investigating visual attention is the aggregation and representation of gaze target positions in a scan path or a superimposed attention map, also commonly referred to as heat map $[6,7,8]$. Scan paths are drawn as linearly connected dot sequences, depicting raw gaze points $(\mathrm{x}, \mathrm{y}, \mathrm{t})$ or a subset of those points that satisfy criteria for labeling as fixations, the relatively stationary component of the eye movement. Heat maps provide a much cleaner depiction of aggregate gaze by combining gaze fixations from multiple viewers. These two representations of gaze target positions are suitable for 2D stimuli, but as eye tracking studies typically comprise a time consuming frame by frame analysis of captured screen recordings with superimposed attention maps, the reduction of $3 \mathrm{D}$ scenarios to $2 \mathrm{D}$ is likely to lead to data loss without assessing the hypotheses on 3D stimuli too [2]. Although, several approaches has been done recently to transfer the notion of attention maps from 2D content to $3 \mathrm{D}$ content $[3,6,7,9]$ they all have targeted only $3 \mathrm{D}$ objects and 3D virtual environments. Beside these, heat maps have received relatively little attention and have remained unchanged since their introduction. Hence, visual gaze analysis in real 3D environments lacks methods and techniques for aggregating attentional representations.

\section{The Study}

In this study, a 3D cave automatic virtual environment (CAVE) has been set up for prototyping the service design of a tourist information office space (see figure 1). A CAVE is an immersive virtual reality environment where projectors are directed to three, four, five or six of the walls of a room-sized cube, allowing the exploration of users' experiences in a more realistic but controlled space. Users' viewing behaviour will be collected with the Tobii [10] mobile eye tracker while experiencing the simulated service. Eye gaze patterns will then be presented on a 3D heat map for understanding customers' experiences while in a service. The purpose of this study is in twofold. First, as Experiential Prototyping lacks quantitative methods for gaining insights in users' experiences, the present study, introduces the use of eye gaze as a direct measurement into users' mental processes. Second, an implementation using Augmented Reality (AR) markers has been proposed for representing customers' 
visual attention with actual distances in a CAVE, while experiencing the service. The result, a 3D attention heat map, is expected to be a valuable tool for Service Prototyping.

\subsection{Apparatus and Participants}

A semi-virtual CAVE of a tourist information office was simulated with 3 large projection screens and real physical objects (an information kiosk, a table with brochures and a TV screen for showing advertisements) (see figure 1). The side screens were projected with a video (including audio) and static images with text variating in information content. The center screen was projected with a dynamic synthetic customer representative showing closed non-verbal behavior (e.g. on the phone or working on the computer).

The CAVE was used as an experience prototyping platform. 5 participants, composed of students and researchers from the University, were recruited for this study. The mobile eye-tracker, developed by Tobii [10], provided the technical basis for an analysis of visual attention during the mobile exploration in the CAVE (see figure 1).

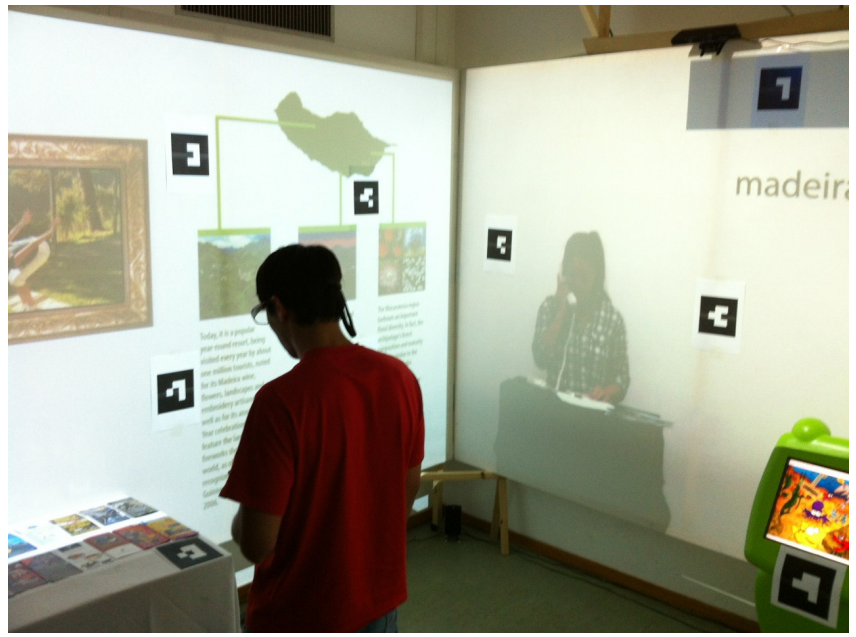

Fig. 1. The simulated tourist information office

\subsection{Augmented Reality (AR) Markers}

Augmented Reality markers are small printable black and white shapes that interact with software downloaded to a computer equipped with a webcam or a smart phone with camera to create $3 \mathrm{D}$ images overlaid on the real world image seen on screen. A total of 16 AR markers were located strategically within the CAVE near visual targets. The set-up was designed so that at least 1 tag was always visible when participants looked through the Tobii Glasses. 


\subsection{Procedures}

All participants were asked to enact a tourist information office visit by going through two tasks, an open task condition and a goal directed task condition. Two different tasks were chosen in order to create the possibility of getting two different types of behavioral approaches and gaze patterns. In the open task condition participants were given an absence of specific plans. In the second task condition they were given a presence of specific plans; they were asked to imagine the following "You heard a tourist talking about their great time during their hike from $\mathrm{x}$ to $\mathrm{y}$. You are interested in this hike as well as other popular hikes and would like to know how to get there, if there is a possibility to camp and any other information about the trail. You also want to know about the possibilities to do whale watching. You have to select one for tomorrow's activity".

Each task, which lasted no longer than 5 minutes, was explained verbally to participants before entering the CAVE. After the task was explained they were asked to enter the CAVE and scan through the environment in order to see what they want to do, see or know more about. All participants were asked to think aloud as much as possible and say out loud as soon as they have decided what they want to approach. This was also an indication of the end of the task. For technical limitations regarding the mobile glasses and AR tags, participants were assigned to first walk to a starting point in the CAVE, indicated with a round symbol on the floor. They were also asked to stay within a particular area when in the CAVE, which was also indicated on the floor.

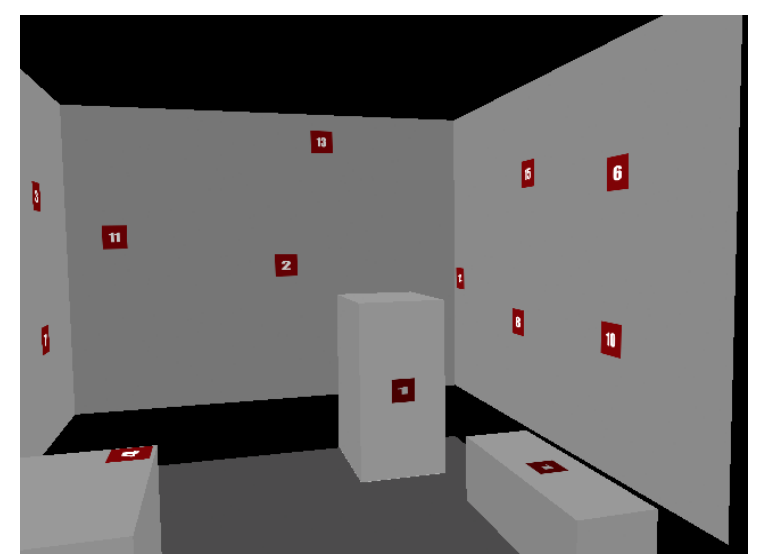

Fig. 2. A visualization of the AR markers in the virtual space

\subsection{Implementation}

After data collection, the first step was to re-create the tourist information office in virtual space using Quartz Composer Development Environment available in Mac OSX, where interactive 3D navigation and real-time visualization is possible. Figure 2 shows the $3 \mathrm{D}$ virtual space of the tourist information office tagged with AR markers, which were used as anchor points for projecting gaze points. Gaze positions were 
converted from 2D into 3D space for each AR marker individually (see figure 3). A video layer was first captured by the scene camera on Tobii Glasses and a gaze layer with eye gaze position was acquired by Tobii Studio Gaze positions in scene camera coordinate system. Since Tobii Glasses is an off-line eye tracker, post-processing is required after experiments. Gaze data and video streams from scene camera were analyzed and exported from Tobii Studio before sending to Quartz Composer for further analysis. Then, the AR markers are detected and recognized in the Quartz Composer. The marker position and rotation in scene camera coordinate system were extracted. The origin was then shifted to the center of the AR marker and the video stream from the scene camera as well as the gaze points are translated to the AR marker's coordinate system. A 3D rotation transformation was performed to adjust the perspective so that the norm vector of the maker is perpendicular to the display. Finally, the eye gaze position was transformed to the appropriate position related to the marker in the virtual tourist office.
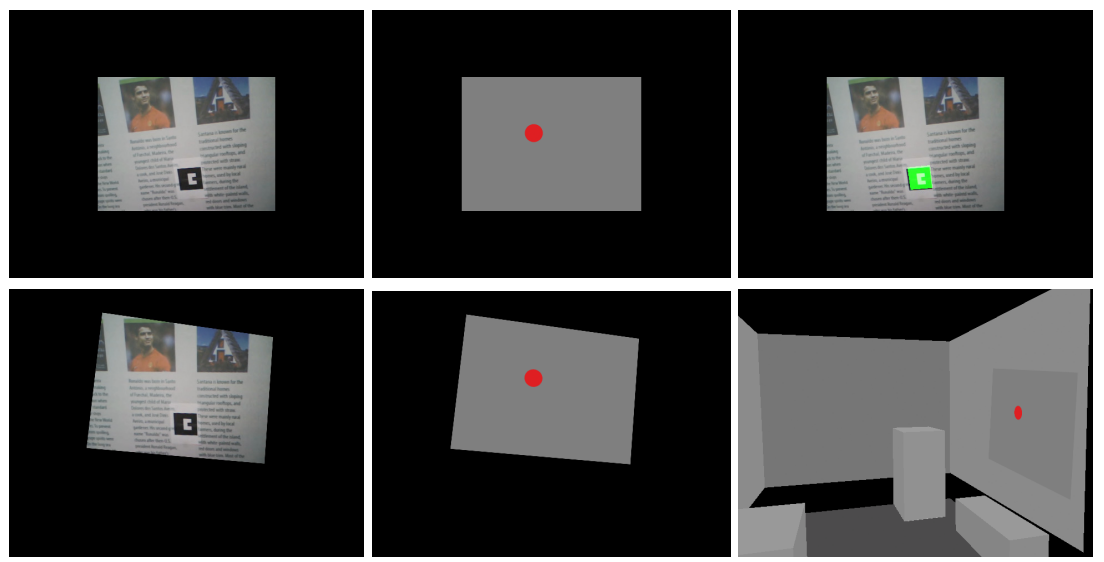

Fig. 3. A video stream captured by the scene camera on Tobii Glasses (top left); A gaze layer with eye gaze position acquired by Tobii Studio Gaze positions in scene camera coordinate system (top middle); AR markers (marker id\# 10) detection and recognition of marker position and rotation in scene camera coordinate system (top right); 3D rotation transformation (bottom left); Eye gaze position transformation to the appropriate position (bottom middle); gaze position converted from the marker coordinate system into the coordinate system of the tourist office (bottom right).

Since the positions of all 16 AR markers in the tourist information office coordinate system were pre-measured in the coordinate system of the tourist office, references were known for calculating gaze points in 3D space. Gaze points are further converted from the gaze position in the marker coordinate system into the coordinate system of the tourist information office. Gaze points were aggregated over the course of the experiment, and across all subjects. The intensity of the luminance represents the frequency of the visual attention. Hence, the "hot" spots in the virtual tourist office indicate areas that attract customers more attention. 


\section{$3 \quad$ Results}

Participants' verbal comments during the think aloud, on the spot observations of participants behaviour and video material were analyzed qualitatively. Overall, in the open task condition, participants seem to divide their attention to different targets within the CAVE in a more balanced manner spending the same amount of time on fixating towards a target as well as moving to other targets. They made more movements and put more effort in trying to cover all information available in the tourist information office.

In the goal directed task condition, participants seem to scan quickly through all targets until they find what they were searching for. They spend more time on fixating toward a target of interest and less time for moving to other targets.

Figure 4 shows the 3D attention heat map of the aggregated eye gaze for all participants separately for both conditions. It seems that in the goal directed task condition, the heat map is more focused and concentrated supporting the qualitative findings. The 3D heat map, however, provides specific details on what has been targeted. The intensity of the heat map reflects the quantity of visual attention of a given position. The by-product of the process provides the access to the raw data of gaze positions in 3D space. With further defining Area of Interests, other quantitative analysis such as fixation count, fixation duration, percentage fixed, etc. could be easily obtained.
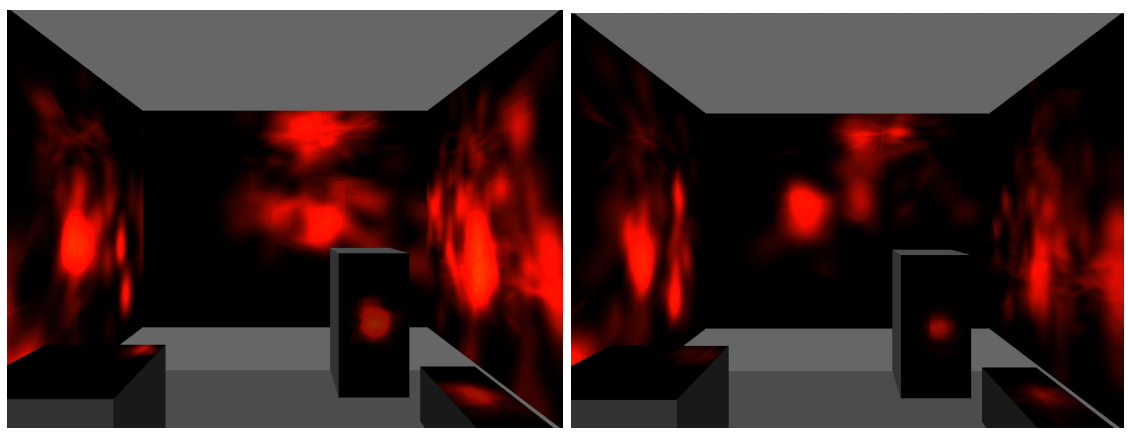

Fig. 4. A visualization of the 3D heat map in the virtual space for the open task condition (left) and goal directed task condition (right)

\section{Discussion and Conclusion}

The interest in exploring and developing experiences in HCI is growing, while often designers of interactive systems go beyond their limits when using tools to explore and communicate what it will be like to interact with the things they are designing. A popular tool to understand, explore or communicate what it might be like to engage with the product, system or space that is being designed is Experience Prototyping. This tool has been of importance in the application of Service Design. Hence, Experience Prototyping is qualitative in nature and characterized with an indirect approach into customers' experience or view towards products or services. Therefore, one of 
the purposes of this study is to introduce a quantitative method for gaining insights in users' experiences, by looking at eye gaze as a direct measurement into users' mental processes.

Although current advancements in mobile eye tracking systems allows the observer to freely interact physically in a 3D scenarios, providing the means to assess eye gaze and visual attention and understand mental processes in arbitrary 3D environments, eye tracking hasn't been applied to Service Design yet. Visual gaze analysis in real 3D environments also seem to lacks methods and techniques for aggregating attentional representations, which directs us to a second purpose of this study; an implementation of representing customers' visual attention with actual distances, while experiencing the service using AR Markers.

A mixed reality CAVE was set up for prototyping the service design of a tourist information office space and users' viewing behaviour was collected with a mobile eye tracker while in a service. Eye movements were recorded and visualized virtually on a 3D attention heat map. The different viewing patterns for different conditions, concludes the 3D heat map to be a valuable tool for prototyping service design. The 3D heat map provides specific details on what has been targeted compared with qualitative methods. The proposed paradigm is independent to the brand, type and model of mobile eye-trackers. Additional efforts can be made to port the software to support other mobile eye-trackers in order to benefit service design community.

Some limitations were found during this study. First, the slow shuttle speed of the scene camera in the eye tracker resulted in blurry images when head movements occurred, which makes it more difficult for AR marker detection. Second, there were problems with the marker distribution within the CAVE. When more markers are used, indicating higher density, the performance improves but obscures the scene. The use of IR markers could be a solution for future studies. In additional to invisible markers, object and scene recognition should be considered as well when tracking in a more realistic scenario where planting markers to cover whole physical space is not feasible. Also, the implementation requires manual operation. Therefore, an eye gaze proxy could be useful once online access to gaze data on the mobile tracker is possible. Future work should focus on more empirical studies to validate the usefulness of the 3D heat maps as well as other new ways of quantitative analysis of visual attention that can be applied to Service Design.

Acknowledgments. The study was done in Logica Service Design Lab with the supports from Knowledge+ incentive system in Madeira, Portugal. We would also like to thank all the volunteers who participated in the study during data collection.

\section{References}

1. Jacob, R.J.K., Karn, K.S.: Eye tracking in Human-Computer Interaction and Usability Research: Ready to deliver the Promises. In: Hyönä, J., Radach, R., Deubel, H. (eds.) The Mind's Eye: Cognitive and Applied Aspects of Eye Movement Research, pp. 573-605. Elsevier Science, Amsterdam (2003) 
2. Pfeiffer, T.: Measuring and Visualizing Attention in Space with 3D Attention Volumes. In: Proceedings of the Symposium on Eye Tracking Research and Applications, March 28-30, pp. 29-36. ACM, New York (2012)

3. Stellmach, Nacke, L., Dachselt, R.: 3D attentional maps: Aggregated gaze visualizations in three-dimensional virtual environments. In: Proceedings of the International Conference on Advanced Visual Interfaces, pp. 345-348. ACM (2010)

4. Stellmach, S., Nacke, L., Dachselt, R., Lindley, C.: Trends and Techniques in Visual Gaze Analysis. In: Proceedings of the 5th Conference on Communication by Gaze Interaction COGAIN 2009: Gaze Interaction for Those Who Want It Most, pp. 89-93. The COGAIN Association, Lyngby (2009)

5. Moritz, S.: Service Design, practical access to an evolving field. Koln International School of Design, London (2005)

6. Pfeiffer, T.: Tracking and Visualizing Visual Attention in Real 3D Space. In: Haack, J., Wiese, H., Abraham, A., Chiarcos, C. (eds.) Proceedings of the KogWis 2010, pp. 220-221. Universittsverlag Potsdam, Potsdam (2010)

7. Spakov, O., Miniotas, D.: Visualization of Eye Gaze Data using Heat Maps. Electronica and Electrical Engineering 2(74), 55-58 (2007)

8. Velichkovsky, B.M., Hansen, J.P.: New technological windows into mind: There is more in eyes and brains for human-computer interaction. In: CHI 1996: Human Factors in Computing Systems, pp. 496-503. ACM Press, NY (1996)

9. Pfeiffer, T.: Understanding Multimodal Deixis with Gaze and Gesture in Conversational Interfaces. Berichte aus der Informatik. Shaker Verlag, Aachen (2011)

10. Tobii Glasses Eye tracker, http: / /www. tobiiglasses . com 